\title{
La maternidad como proyecto individual y autónomo. El caso de las madres solas por elección
}

\author{
María Laura Giallorenzi ${ }^{1}$ \\ CONICET- UNR
}

\section{Artículo científico}

Material original autorizado para su primera publicación en el Journal de Ciencias Sociales, Revista Académica de la Facultad de Ciencias Sociales de la Universidad de Palermo.

Recepción: 15-09-2018

Aceptación: 24-09-2018

Resumen: El presente artículo tiene como propósito socializar parte de una investigación que se enmarca en una tesis doctoral en Ciencias Sociales financiada por una beca doctoral del Consejo Nacional de Investigaciones Científicas y Técnicas radicada en la Universidad Nacional de Rosario. La temática concierne al análisis de la maternidad en solitario y por elección, dentro de un escenario de transformaciones para el rol de la mujer y de nuevos contextos de filiación en la actualidad de la Argentina. El objetivo general de este trabajo es analizar de manera exploratoria las particularidades que caracterizan actualmente a la maternidad en solitario y por elección en el marco de las transformaciones del rol de la mujer y de los nuevos contextos de filiación. En primer lugar, se sitúa esta forma de maternidad dentro de un contexto de transformaciones políticas, culturales y en materia legislativa, que permite describir su especificidad. En segundo lugar, se realiza un breve recorrido por la teoría feminista con el fin de analizar la categoría de maternidad y de mujer a lo largo de las últimas décadas. Por último, se presentan los primeros resultados obtenidos a partir del análisis de entrevistas semiestructuradas realizadas a madres solteras por elección.

Palabras claves: maternidad; transformaciones; rol de la mujer; filiación.

\section{Maternity as an Individual and Autonomous Project. The Case of Single Mothers by Choice}

Abstract: This paper aims to socialize part of a research that is in the context of a doctoral thesis in Social Sciences funded by a Doctoral Scholarship of the National Council of Scientific

\footnotetext{
${ }^{1}$ Becaria Doctoral de CONICET, Doctorado en Ciencias Sociales (UBA), Magister en Estudios Culturales (UNR), Licenciada en Sociología (UCES), Profesora Universitaria (UCEL). Profesora titular en la Universidad de Concepción del Uruguay sede Rosario y adscripta en la Facultad de Ciencias Políticas y Relaciones Internacionales de la Universidad Nacional de Rosario. Correo electrónico: giallorenziml@yahoo.com.ar
} 
and Technical Research located in the National University of Rosario. The theme is about the maternity analysis acting alone and by choice, within a scenario of transformations for the role of women and new filiation contexts in the current Argentine society. The overall purpose of this work is to analyze in an exploratory way the particularities that currently characterize maternity acting alone and by choice within the framework of the transformations of the role of women and of the new filiation contexts. In the first place, this maternity way is placed in a context of political, cultural and legislative transformations, which describe its specificity. Secondly, a brief study of feminist theory is carried out in order to analyze the maternity category and womanhood over the last decades. Finally, the first results obtained from the analysis of semi-structured interviews conducted to single mothers by choice are presented.

Keywords: maternity; transformations; role of women; filiation.

\section{Introducción}

El campo del problema de esta investigación concierne al análisis de la maternidad en solitario y por elección, dentro de un escenario de transformaciones para el rol de la mujer y de nuevos contextos de filiación en la actualidad. Puntualmente, para este artículo, el análisis estará estructurado sobre la base de los siguientes ejes: la decisión de ser madres, de ser madres solas por elección, cómo eligieron la forma para llevarlo a cabo y cómo llevan a cabo su maternidad en relación con la crianza y el cuidado.

La novedad del caso analizado no reside en que estas mujeres se hagan cargo en solitario de la crianza de sus hijos/as, sino en la particularidad de que la maternidad expresa un proyecto individual y autónomo que se presenta escindido de la pareja y la conyugalidad.

La comunidad científica las ha designado como Madres solas por elección (MSPE), del inglés Single mothers by choice, un epígrafe en el que se incluyen las mujeres que eligen -al menos, inicialmente- ser madres solas sin una pareja que comparta con ellas la concepción, el embarazo y/o la crianza de los/as hijos/as, y a partir de ello recurren a diversas estrategias y procedimientos para lograrlo (adopción, técnicas de reproducción asistida, acuerdos con terceros, etc.). Por medio de la apropiación de este epígrafe, "las mujeres reclaman implícitamente el derecho a tomar esa decisión en solitario, utilizando para ello diversos argumentos que doten de legitimidad su decisión y su forma familiar" (Bock citado en Frasquet, 2013, p. 164).

Las madres solas por elección protagonizan una forma de maternidad caracterizada por la decisión propia y no por algún tipo de abandono o suceso vital, sino que esa soledad expresa el desencanto que experimentan respecto al modelo de familia biparental y con ello la afirmación de la propia autonomía individual, del derecho al propio cuerpo y a la maternidad 
(Frasquet, 2013; Jociles y Rivas 2009 y 2010; Jordana Pröpper, 2011). La monoparentalidad elegida con respecto a aquella que no fue buscada expresa la disociación del proyecto de la maternidad del de emparejamiento (pareja, matrimonio, etc.), y evidencia la construcción de la maternidad como un proyecto autónomo e individual.

Es necesario aclarar que quedan excluidas de esta forma de maternidad aquellas mujeres que deben llevarla en solitario a partir de una situación de viudez, abandono, separación o divorcio. Los estudios previos realizados en España sobre la temática (González, Díez, Jiménez, Morgadhan, 2004 y 2008; Moncó, Jociles, Rivas, 2011) reservan la denominación de madres a solas para este último grupo. La razón por la cual este grupo no se incluye en el caso de las madres solas radica en que aquí no se realiza una elección a priori respecto de la forma en que se llevará a cabo la maternidad.

El objetivo general de esta investigación es analizar de manera exploratoria las particularidades que caracterizan actualmente a la maternidad en solitario y por elección en el marco de las transformaciones del rol de la mujer y de los nuevos contextos de filiación.

En este sentido, la maternidad ha sido analizada en sus múltiples expresiones como la maternidad lesboparental (Donosso, 2013; Lewin, 1995), la maternidad subrogada (Smietana 2013; De La Barreda, 2017), la maternidad adoptiva (Cadoret, 2009; Fonseca, 1998; Gross, 2009), la maternidad por técnicas de reproducción asistida (Bestard, 2011), la maternidad en mujeres trans (Álvarez-Díaz, 2009) y la maternidad en solitario y por elección (Frasquet, 2013; González, Díez, Jiménez, Morgadhan, 2004 y 2008; Moncó, Jociles, Rivas, 2011; Jordana Pröpper, 2011). Todas estas maneras de experimentar la maternidad tienen en común el hecho de que la filiación biológica, jurídica, social y afectiva ya no coinciden necesariamente entre sí.

Se parte del supuesto de que a lo largo de la historia se fomenta a las mujeres a que sean madres, pero no de manera solitaria. Los modelos obligatorios como la maternidad implican en su definición también las formas de llevarlos a la práctica (Juliano, 2004; Frasquet, 2013).

A partir de este supuesto, se partió de dos hipótesis de trabajo que han orientado una primera etapa exploratoria y descriptiva de la investigación: las mujeres que eligen ser madres solas por elección desafían los mandatos sociales de género y modelos tradicionales de maternidad y familia. $Y$ la elección de ser madre sola de parte de mujeres adultas y económicamente independientes es un reflejo de procesos sociales de marcada tendencia hacia la autonomía económica, social y afectiva. Ya en una segunda etapa, y a partir del trabajo de campo, se cuenta con una nueva hipótesis: la elección de ser madres solas por parte de mujeres adultas e independientes es un indicio de que la concreción de la maternidad se encuentra modificada por la individualización social y por la aparición de técnicas de 
reproducción asistida y la adopción monoparental que reforzarían el ideal de que las mujeres deben ser madres.

Esta investigación, y la especificidad de su contexto, pueden situarse a partir de las décadas de 1970 y 1980 como efecto de una intensa campaña iniciada por movimientos de mujeres en los países industrializados, donde se producen cambios en la agenda de muchos gobiernos que propician avances en materia de derechos reproductivos y fundan el escenario propicio para que estos nuevos contextos de filiación se inscriban. Así, los derechos reproductivos pasan a ser reconocidos como derechos humanos básicos, ligados entre otros al derecho a la salud y especialmente referidos al derecho a decidir libremente si tener hijos o no, cuántos y cuándo tenerlos (Suárez Llanos, 2002; Dorlin, 2009).

En Argentina, los avances en materia de derechos reproductivos se expresan recién en el año 2013, con la sanción de la ley № 26.862 de Acceso integral a los procedimientos y técnicas médico-asistenciales de reproducción médicamente asistida, reglamentada a través del Decreto 956/2013. Esto permite que muchas mujeres puedan planificar su maternidad a través del acceso a bancos de gametos masculinos y/o postergar su maternidad a partir del congelamiento de óvulos. Tanto la puesta en vigencia de esta ley como la cobertura de las obras sociales y el avance en tecnología médica posibilitan de manera conjunta que la maternidad acontezca de maneras muy diferentes a como se daba en años previos.

Es por ello que, para esta investigación, se toma como caso a analizar las mujeres que para convertirse en madres solas han recurrido a un tratamiento de fertilización asistida o a la adopción monoparental.

Se propone realizar un estudio colectivo de caso (Stake, 1999) tomando como unidad de análisis a madres solas por elección de la República Argentina.

Se opta por otorgar el estatuto de caso a las madres solas por elección, dado que se trata de la materialización de una de las múltiples formas de ser madre en la actualidad. Se espera que el estudio en profundidad de este caso funcione como punto central de un análisis más amplio que permita poner en relación esta forma de maternidad con las transformaciones que vienen aconteciendo en relación con el rol de la mujer en la actualidad.

Con esta estrategia metodológica de carácter cualitativo se pretende acceder, por lo tanto, a una "comprensión más precisa" (Stake, 1999, p. 20) de un caso particular -como es la maternidad en solitario y por elección-, para entender la situación más general del rol de la mujer en la actualidad, llevada adelante con las herramientas conceptuales de las teorías feministas.

Por lo tanto, el cometido de esta investigación, como ocurre en todo estudio de caso, es la particularización y no la generalización. Se pretende conocer bien el caso particular de 
las madres solas por elección, no para ver en qué se diferencia respecto de las otras formas de maternidad, sino para indagar en su unicidad, renunciando de antemano a toda generalización. Esto implica la priorización de "la unicidad del caso" (Stake, 1999, p. 20) por sobre su representatividad.

Si bien puede plantearse la maternidad en solitario y por elección como un caso, existen numerosas mujeres que entran en este grupo. Es por ello que se realiza un estudio instrumental (Stake, 1999), el cual permite elegir una unidad de análisis por la conveniencia que esta presenta respecto de otras posibles. A su vez, dada la cantidad de mujeres que son en la actualidad madres solas por elección, y que cada una de ellas presenta a su vez características que pueden diferir de las otras, se propone realizar un estudio colectivo de casos, el cual consiste en elegir una serie de elementos dentro de un conjunto mayor.

En un primer momento de la investigación se realiza una indagación bibliográfica para situar la forma en que las teorías feministas plantean el rol de la mujer, otorgando un lugar prioritario a las principales críticas que han realizado sobre ello. Asimismo, se recorren las posturas que algunas de estas autoras han tomado frente a las formas de ser madre en la actualidad.

Por otro lado, esta indagación se orienta a situar los elementos clave de los factores sociales, económicos, culturales, jurídicos y tecnológicos que podrían estar contribuyendo actualmente a la emergencia de esta forma de maternidad. Para completar esta indagación bibliográfica, se realizan entrevistas a informantes clave como médicos/as especialistas en reproducción asistida y abogados/as especialistas en adopción.

En un segundo momento de la investigación, se realizan entrevistas semiestructuradas y en profundidad, con la finalidad de conocer en detalle el caso. Con el objetivo de indagar el problema que interesa, se pretende realizar un número acotado de entrevistas a mujeres que sean madres solas por elección en el presente, o bien que estén iniciando este proyecto de familia.

La guía de pautas está diseñada en torno a algunos ejes que resultan relevantes a partir de estudios previos sobre la temática y a un primer acercamiento a la población a entrevistar: experiencia de maternidad, el proceso de toma de decisiones, el modo de acceso a la maternidad, la conciliación familia-trabajo (esferas productiva y reproductiva), la visión de la sociedad o la valoración de la experiencia de maternidad.

La selección de los sujetos a entrevistar se realiza por conveniencia, dadas las características del caso, y su número estará delimitado por la saturación teórica (Sautu, 2005). Dado que los datos obtenidos no siempre presentarán características comunes, se contempla incluir toda la multiplicidad de voces que se manifieste en las entrevistas. Es decir, 
los sujetos a entrevistar interesan "tanto por lo que tienen de único como por lo que tienen de común" (Stake, 1999, p. 15).

Se opta por la técnica de la entrevista porque constituye un valioso recurso para que los sujetos a entrevistar transmitan sus vivencias de la manera más directa y explícita posible. Dentro de esta, se elige la entrevista semiestructurada, entendiéndola como un conjunto de preguntas y cuestiones básicas a explorar, pero donde la redacción y el orden de preguntas no se encuentran determinados (Valles, 1999). Las entrevistas están organizadas sobre la base de los siguientes ejes de análisis: la experiencia de maternidad, el proceso de toma de decisiones, la crianza y el cuidado como categoría de parentesco y la valoración de terceros sobre la experiencia de maternidad.

\section{La maternidad desde la teoría feminista}

El marco teórico que se presenta a continuación tiene como conceptos centrales la noción de maternidad y la materialización de las mujeres. Para ello, se han elegido algunas autoras que han teorizado sobre ellos desde la teoría feminista.

Dentro de la teoría feminista, se ha problematizado de manera crítica las construcciones y los efectos de los diversos discursos sociales sobre las mujeres, entre los que se destacan los discursos sobre la maternidad (Badinter, 1991; Rich, 1986; Friedan, 2009; Beauvoir, 2012, entre otras).

La noción moderna de maternidad y la carga valorativa implícita en la idea de buena madre comienza a fraguarse en el último tercio del siglo XVIII. Según Badinter (1991), es a partir de 1760 que se produce la revolución conceptual de la maternidad, asociándola a ciertas imágenes y funciones que se incorporan a las mentalidades y conductas de la época. Esto coincide con la emergencia y el desarrollo del capitalismo y con la transformación de la familia como unidad económica y social encargada de ocuparse de la supervivencia de los niños. Se evidencia así la estrecha relación existente entre el discurso económico de la época -apoyado en la creación de una nueva ciencia como la demografía- con fundamentos de orden social y cultural dirigidos a las familias y a las mujeres en particular (Badinter, 1991; Darré, 2013).

En este contexto, los discursos de la época encarnados en instituciones disciplinares "le crean a la mujer la obligación de ser ante todo madre, y engendran un mito que doscientos años más tarde seguirá más vivo que nunca: el mito del instinto maternal, del amor espontáneo de toda madre hacia su hijo" (Badinter, 1991, p. 79).

De esta manera, comienza a fortalecerse una asociación inédita hasta el momento entre los términos amor y maternidad, que implica no solo la promoción social de este 
sentimiento humano sino también la inseparable unión del mismo a la mujer en tanto madre: amor-maternal, no serán, a partir de allí, dos elementos de un binomio, sino la nominación de un sentido indisoluble.

Así, las trasformaciones destinadas a "preparar a una mujer para este rol y convencerla de que tener hijos y marido es lo mejor que puede esperar de la vida" (Federici, 2013, p. 37), fueron promovidas principalmente por el capitalismo a partir de la institucionalización de diversas disciplinas -el saber teológico y médico en un comienzo, y luego la Demografía, la Psicología, entre otros- encargadas de justificar y regular el orden poblacional que el nuevo modelo económico requería. En este marco, la configuración de una estructura familiar, tal como se conoce en el presente, se convirtió en garante de producir la mano de obra continua que el sistema necesitaba para su consolidación.

Pero no todas las mujeres en todas las regiones se vieron afectadas por las mismas condiciones, y la evolución de las costumbres fue más lenta de lo que se esperaba. Ciertamente, la figura de la nueva madre fue pregonando más rápidamente en las clases trabajadoras y los sectores medios urbanos, así como en la burguesía acomodada de los países industriales desde fines del siglo XVIII. Como se expresa en la siguiente cita:

Las mujeres más cumplidas en su condición de madres aceptaron gozosamente cargar con ese peso temible. Para ellas la empresa valía la pena. Pero las demás, más numerosas de lo que cabría creer, no pudieron tomar distancia respecto del nuevo papel que se las obligaba a desempeñar sino a costa de angustia y de sentimiento de culpa. La razón es simple: quienes definieron la 'naturaleza femenina' tuvieron cuidado de hacerlo de manera tal que implicara todas las características de la buena madre. (Badinter, 1991, p.131)

Este proceso de maternalización de las mujeres también ha sido fuertemente revisado por Simone de Beauvoir (2012) en su clásico y consagratorio libro El Segundo Sexo publicado en el año 1949, donde reflexionó sobre la configuración histórico-social de la maternidad como destino ineludible para todas las mujeres:

En virtud de la maternidad es como la mujer cumple íntegramente su destino fisiológico; ésa es su vocación "natural", puesto que todo el organismo está orientado hacia la perpetuación de la especie. Pero ya se ha dicho que la sociedad humana no está jamás abandonada a la Naturaleza. Y, en particular, desde hace aproximadamente un siglo, la función reproductora ya no está determinada por el solo azar biológico, sino que está controlada por la voluntad. (p. 464)

De su famosa obra se ha dicho que, hasta ese momento, nadie había expuesto de manera tan profunda y sencilla la idea de que "no se nace mujer, se llega a serlo" (Beauvoir, 2012, p. 207). Con esta frase, de Beauvoir separa la naturaleza biológica de la cultura y da 
lugar al desarrollo posterior de la categoría de género como construcción social. Pero, fundamentalmente, lo que la escritora va a denunciar es el reinado de la domesticidad obligatoria de las mujeres en sus roles de buenas esposas y buenas madres (Varela, 2005).

Para esta autora, desde la infancia las hembras humanas son condicionadas por discursos que refuerzan la idea de estar hechas para engendrar, apelando a los sentimientos conmovedores producidos en y por la maternidad. También se le advierte sobre los inconvenientes de las condiciones propias de su sexualidad -menstruación, dolores, enfermedades, etc.- y sobre el tedio de las tareas domésticas. Pero todo ello "queda justificado por ese maravilloso privilegio que ostenta traer hijos al mundo" (Beauvoir, 2012, p. 473).

Más tarde, la crítica feminista, radicalizada en los años '60 en Estados Unidos y Europa con diferencias y matices, se ha considerado heredera del libro que convirtió en feminista a la propia Simone de Beauvoir (Varela, 2005)².

Es a partir del concepto de situación que la autora da cuenta de los inconvenientes con las que se encuentra una mujer para poder ser un sujeto, libre y autónomo. Así, son esas dificultades las que van a explicar la sujeción de la mujer a la vida biológica, a la maternidad y a la reproducción, las cuales son concebidas como exclusivamente biológicas, a la vez que se niega su contenido social y cultural. En este sentido, de Beauvoir (2012) afirma:

Tal obligación no tiene nada de natural: la naturaleza jamás podría dictar una elección moral; esta implica un compromiso. Parir es adquirir un compromiso; si la madre lo rehúye después, comete una falta contra la existencia humana, contra una libertad; pero nadie puede imponérselo. (p. 509)

Por tanto, en la medida en que se piensa que para que una mujer pueda lograr trascenderse debe arriesgar la vida biológica, desde su perspectiva la liberación de las mujeres y su constitución como sujetos trascendentes acontece por la negación del cuerpo, ámbito de la inmanencia.

Ya se ha visto que la inferioridad de la mujer procedía originariamente de que, en principio, se ha limitado a repetir la vida, mientras que el hombre inventaba razones para vivir, más esenciales a sus ojos que la pura ficción de la existencia; encerrar a la mujer en la maternidad seria perpetuar esa situación (Beauvoir, 2012, p. 511)

\footnotetext{
2Para Celia Amorós, buena parte del feminismo de la segunda mitad del siglo XX, o todo, puede ser considerado comentarios o notas al pie de página de El segundo sexo y para Teresa López Pardina, "este famoso ensayo marca un hito en la historia de la teoría feminista, no sólo porque vuelve a poner en pie al feminismo después de la Segunda Guerra Mundial, sino porque es el estudio más completo de cuantos se han escrito sobre la condición de la mujer" (López Pardina, 1999, p.18).
} 
En este sentido, la maternidad es una alienación, un proyecto inauténtico que se ofrece a las mujeres evitando que se realicen como trascendencia. Entonces para de Beauvoir, las mujeres solo alcanzarán la plena humanidad cuando abandonen esa situación de opresión y elijan el camino de la trascendencia (del cuerpo), el camino de la emancipación de los hombres.

Recapitulando, El segundo sexo cala con profundidad en una nueva generación de mujeres que, luego de la Segunda Guerra Mundial, ya habían conseguido el acceso al voto y ciertos avances en los derechos educativos. Entre estas mujeres, se destacan figuras como Betty Friedan que, en 1963, publica su célebre ensayo La mística de la femineidad, donde desarrolla una minuciosa caracterización de un malestar generalizado, un problema sin nombre. Se refiere al padecimiento que abarcaba a miles de mujeres que, como ella, sentían una profunda insatisfacción consigo mismas y con la vida en el contexto del desarrollo del Estado de Bienestar y la sociedad de consumo posbélica. Así lo explicará luego en su autobiografía:

Lo que de verdad quería, era ser una ama de casa feliz y realizada, afincada en un barrio residencial y muy pronto madre de tres hijos. Pero recuerdo que un domingo que salimos de excursión con la familia y luego, otra vez, en el aparcamiento de un supermercado, sentí un ataque de pánico repentino, inexplicable, aterrador. Aquello era peor que el asma. (Friedan, 2003, p. 103)

Friedan profundizó los postulados de de Beauvoir revisando sus propias sensaciones, y las de las mujeres que la rodeaban. Pudo advertir que aquellas que valoraban más su educación, que se mostraban alegres y positivas con respecto a su vida eran las que no encajaban exactamente con el rol de "esposa, madre, ama de casa, entregada a su marido, a sus hijos, al hogar, mientras que las que manifestaban dedicarse exclusivamente a esos roles estaban deprimidas (medicadas) o totalmente frustradas" $(2003, \text { p. } 137)^{3}$.

Su libro, que también se convirtió en un clásico del feminismo en los primeros años de la década de 1960, será criticado por otras representantes del movimiento que comenzaban a politizar más radicalmente sus posturas y definiciones al calor de las luchas sociales que se gestaban en la misma década. Las principales críticas a Friedan sostenían que esta se centraba solo en la realidad de las mujeres de la clase media en Estados Unidos y no daba una teoría explicativa del patriarcado como sistema de legitimación del orden social y sustentador de los privilegios masculinos (Varela, 2005).

\footnotetext{
${ }^{3}$ Su particular experiencia de vida y su formación en psicología social la llevaron a descifrar el rol opresivo y asfixiante del modelo ama-de-casa-madre-de-familia que se imponía como obligatorio a las mujeres de medio mundo, generando malestar y descontento femenino. Frente a esta situación se preguntó por: '¿qué hacía que la mística pareciera inevitable, absolutamente irreversible y que cada mujer pensara que estaba sola ante "el problema que no tiene nombre", sin darse cuenta jamás que había otras mujeres a las que no les producía el menor orgasmo sacar brillo al suelo del cuarto de estar?' (Friedan, 2009, p. 137).
} 
Finalmente, los feminismos posteriores le cuestionaran tanto a Friedan como al feminismo liberal y reformista que esta representaba, la inconsistencia de definir la situación de la mujer como desigualdad y no como opresión o explotación (De Miguel, 2000). No obstante, esta mirada facilitó que se impulsaran reformas tendientes a incluir a las mujeres en la esfera pública y en el mercado laboral. Sin dudas, las propuestas del feminismo liberal han sido las más metabolizadas por los sistemas estatales y esta tendencia reformista se ha extendido con cierto éxito hasta la actualidad ${ }^{4}$.

Sin embargo, en los años '70, los principios feministas liberales mostrarían rápidamente sus límites y muchas de las hijas del segundo sexo se acercarán a posturas políticas más radicales con una fuerte raíz socialista (Varela, 2005).

Esto creó una atmósfera propicia al re-encendido del debate sobre la maternidad y la familia. La interpelación al determinismo de la matricentricidad está presente en obras como la de Adrienne Rich (1986) que propone distinguir aspectos de la maternidad en tanto institución o experiencia, en su célebre obra Nacemos de mujer. Allí, la autora plantea que la maternidad se sustenta mediante la superposición de dos sentidos: "la maternidad como la relación potencial de cualquier mujer con su capacidad de reproducción y con los hijos; y la institución cuyo objetivo es asegurar que este potencial -y todas las mujeres- permanezcan bajo el control masculino" (p. 47).

Para ello retoma la discusión realizada por sus antecesoras ${ }^{5}$ sobre la falacia del instinto maternal que podemos encontrar en la siguiente cita: "la maternidad institucionalizada exige de las mujeres 'instinto' maternal en vez de inteligencia, generosidad en lugar de auto realización, y atención a las necesidades ajenas en lugar a las propias" (p. 85) (las comillas son de la autora). De esta manera, Rich advierte sobre las formas ocultas de socialización y las presiones que abiertamente empujan a las mujeres hacia el matrimonio y el amor heterosexual donde "la maternidad institucionalizada revive y renueva todas las demás instituciones" (1986, p. 89).

Hasta aquí hemos procurado presentar algunas líneas interpretativas sobre la noción de maternidad y el proceso de maternalizacion de las mujeres, recorriendo los aportes

\footnotetext{
${ }^{4}$ Unas de las medidas más significativas impulsada por esta corriente fueron las leyes de cupo y sus principios reformistas impregnaron la mayoría de las declaraciones universales emanadas de la época que se inicia con la declaración de 1975 como Año Internacional de la Mujer por las Naciones Unidas. Cinco años más tarde, en 1980, Copenhague fue sede de la Conferencia Mundial del Decenio de las Naciones Unidas para la Mujer, celebrada bajo el lema de Igualdad, desarrollo y paz, y centrada en los temas de salud, educación y empleo (De Miguel, 2000).

5 Una de las referencias ineludibles para la autora es la propuesta integral de Firestone (1976) elaborada en $\mathrm{La}$ dialéctica del sexo, que amplía la definición de materialismo histórico de Engels, colocando la división biológica de los sexos con fines reproductivos como origen de la división misma de clases y define desde allí la perspectiva materialista de la dialéctica sexual, la cual organiza las relaciones entre las clases sexuales. Para Firestone, en la perspectiva dicotómica de los componentes 'varón'-'mujer', el 'trabajo reproductivo' ocupa un lugar nodal, por ser la reproducción biológica y las funciones derivadas de ella, el eje sobre el que descansa la opresión de las mujeres que garantiza el 'trabajo productivo' (Laudano, 2013, p. 3).
} 
teóricos que se consideran más relevantes, emanados de corrientes de pensamiento feministas que, como hemos intentado exponer, se articulan, en gran medida, con las luchas políticas y procesos históricos que dieron lugar a sus reflexiones, debates y reivindicaciones.

\section{Metodología y resultados}

A continuación, se especifica el planteo metodológico realizado, la construcción del caso a estudiar y los resultados parciales obtenidos.

Se explicita que se realiza un estudio colectivo de caso (Stake, 1999) y se tomó como unidad de análisis madres solas por elección que residan en grandes ciudades de nuestro país. La unidad de análisis tomada está formada por mujeres de 35 a 45 años de edad, con estudios terciarios y/o universitarios y que sean jefas de hogar.

La segmentación realizada se basa en dos aspectos: en cuanto al rango etario, el cual fue establecido teniendo en cuenta una edad productiva en que las mujeres que compongan la muestra puedan haber terminado sus estudios universitarios y establecerse laboralmente en el mercado. Su límite superior está dado por la mayoría de edad que impone la Sociedad Argentina de Medicina Reproductiva para acceder a un tratamiento de fertilización asistida. El segundo aspecto que se tuvo en cuenta en el armado de la muestra estuvo dado por aquellas características que permitieran ser indicadores de las transformaciones para el rol de las mujeres como ser el nivel educativo y la residencia urbana donde se tiene un mayor acceso a los puestos laborales de mayor jerarquía.

Se otorga el estatuto de caso a las madres solas por elección, dado que se trata de la materialización de una de las múltiples formas de ser madre en la actualidad. Se espera que el estudio en profundidad de este caso funcione como punto central de un análisis más amplio que permita poner en relación esta forma de maternidad con las transformaciones que vienen aconteciendo en relación al rol de la mujer en la actualidad.

Como parte de los resultados de la investigación, se han seleccionado 6 para este artículo algunas de las entrevistas realizadas hasta el momento para mantener una muestra representativa a madres solteras por elección. Las 4 entrevistas seleccionadas fueron realizadas a mujeres que ya son madres solteras por elección, dos de ellas por adopción y dos por técnicas de fertilización asistida.

Las mujeres entrevistadas cumplieron con las siguientes características sociodemográficas: carrera universitaria, trabajo jornada completa, residencia en grandes

\footnotetext{
${ }^{6}$ Las 4 entrevistas utilizadas para este artículo fueron seleccionadas de un total de 10 entrevistas que constituyen -hasta el momento- el estudio colectivo de caso.
} 
ciudades (Rosario y Capital Federal) y rango de edad de 35 a 45 años. El proceso de selección es a partir de bola de nieve.

Asimismo, se han entrevistado a dos médicas especialistas en fertilización asistida con el objetivo de comprender en detalle algunos de los procedimientos técnicos que relataban las entrevistadas en sus testimonios.

Para este artículo, se analizarán algunas cuestiones en particular como ser: la decisión de ser madres, de ser madres solas por elección, cómo eligieron la forma para llevarlo a cabo y cómo llevan a cabo su maternidad en relación a la crianza y el cuidado.

Las familias lideradas por mujeres que deciden y planifican una maternidad en solitario son una realidad social y cultural que emerge desde hace un tiempo en nuestras sociedades occidentales. Como ya se mencionó, la novedad del caso no reside en que estas mujeres lleven a cabo su maternidad en solitario sino en la planificación de la maternidad como un proyecto autónomo e independiente de la conyugalidad. Así, se expresa como la culminación de un deseo largamente buscado que se constituye como un elemento identitario, tal como lo afirma una de las entrevistadas:

Lo intenté siempre, tener una familia, siempre estuve de novia y noviazgos largos, para lo que va hoy en día, larguísimos, porque hoy nadie está de novio, pero bueno. Lo intente siempre y no se dio. Siempre yo quería lo otro, de hecho te digo que uno de los fracasos tal vez fue el querer formar una familia y que la persona que estaba conmigo en ese momento no quiera. Pero la idea siempre seguía estando, pasaron los años, como que yo ya estaba descreída un poco de esto de la pareja porque no se daba y yo ponía todo, y llore un montón, me enamore un montón, entonces dije esto otro lo quiero de siempre y bueno me inscribí en el 2011 en un juzgado como postulante de adopción. (Cayetana, profesional, 44 años, hija por adopción.)

Siempre quise ser madre. Lo de madre sola se dio porque yo estaba sola y llego el momento en que tenía que darle para adelante por el tema de la edad y no sabía si iba a quedar en la primera, y bueno y quede a los 32 años. Yo siempre quise ser mamá, en realidad yo estaba en pareja con una chica y lo que yo plantee es que yo iba a ser mamá y si ella quería estar, pero el plan era que seamos nosotras dos y nada más (hija y madre). (Analía, técnica, 44 años, hija por fertilización asistida.)

Como se ve, la maternidad se constituye como un deseo propio que se trae desde antes de la conformación de una pareja y que persiste en el tiempo aun cuando no se logre la conformación de una familia biparental. Otros elementos se suman a la toma de decisión por parte de quienes eligen y planifican su maternidad en solitario, la edad y el límite biológico para ser madre. Así lo expresan las entrevistadas: 
A los 27 años, ponele de mi edad, ahí me nació el ser madre y no otra cosa y dije bueno a los 30 es mi límite, a los 30 voy a tener un hijo como sea, así que fui a averiguar. (Silvana, profesional, 41 años, hijo por adopción.)

Me cansé de frustrarme... entonces dije bueno antes de los 40 no voy a dejar pasar el tiempo y voy a ser madre. (Verónica, profesional, 39 años, hijo por fertilización asistida.)

Por un lado, se puede ver como en el relato aparecería una coincidencia entre el deseo de ser madre y el periodo fértil femenino. Esta asociación, que se plantea como algo individual promovido por el deseo interior, es pensada desde las teorías feministas como el resultado de una construcción social y cultural del sistema patriarcal que, a partir de múltiples dispositivos educativos y culturales, le ha inculcado a la mujer la obligatoriedad de ser madre para afirmar su condición de mujer (Rich, 1986; Beauvoir, 2012; Federici, 2013).

Además, en el segundo testimonio aparece el condicionamiento del límite de la edad biológica para aquellas mujeres que desean ser madres por un tratamiento por fertilización.

Para quienes realizan un tratamiento de fertilización asistida, la posibilidad de llevar a cabo la maternidad de manera biológica, posibilitador de la experiencia del embarazo, el parto y la lactancia, adquiere valor al reafirmar ante el imaginario social los componentes que históricamente han sido asociados a la identidad femenina (Lamas, 2002; Schwarz, 2016). Así lo menciona Verónica en su relato:

Así como tenía la idea de ser madre siempre tuve la idea de que adoptar no. Si era mío porque Dios me lo mandaba porque tenía que ser, iba a hacer todo lo posible para tener un hijo, pero adoptado no. Yo quería estar embarazada. (Verónica, profesional, 39 años, hijo por fertilización asistida.)

Aunque en las familias monoparentales la maternidad no ocurre naturalmente ya que estas mujeres deben recurrir a la donación de gametos masculinos, la conexión biológica adquiere valor relacional y funda las relaciones de parentesco. En esta reelaboración simbólica, la biología ha sido desplazada de su centralidad y se impone la intencionalidad como símbolo central de la categoría de parentesco (Smietana, 2013; Frasquet, 2013).

Por otro lado, la adopción aparece como una alternativa para aquellas mujeres que definen a la maternidad "en términos de relación social, concediendo mayor valor a la filiación social que a la filiación genética" (Donoso, 2013, p. 193). En este sentido, el lugar de lo biológico y con ello el embarazo, el parto y la lactancia ya no son exclusivos de un proceso necesario para convertirse en madres, otorgándole una valoración mayor a la idea de realizar una acción predominantemente altruista. Así lo afirman dos de las entrevistadas en sus testimonios: 
Es un deseo que yo tengo y hay alguien que necesita algo. Entonces me parece más copado, más noble, más amoroso, más generoso y no de egoísmo. Entonces como que me cerraba de todos lados la idea de la adopción, este... Hay alguien que no tiene y yo estoy acá para darlo. (Cayetana, profesional, 44 años, hija por adopción.)

Vos al traer a alguien al mundo traes una vida más al mundo, otra persona que sigue consumiendo de este mundo y habiendo tantos otros que ya están en disponibilidad, consumiendo y viviendo en este mundo, ellos con su necesidad y yo con mi deseo, es como que una cosa y la otra se unen y salió algo hermoso. Tener otro hijo, traer otro más, no sé... (Silvana, profesional, 41 años, hijo por adopción.)

En ambos casos la elección y la intención de ser madres dan origen a la relación de filiación, donde la crianza y el vínculo afectivo constituyen elementos suficientes para construir relaciones de parentesco. Es la capacidad de agencia de las mujeres, el trabajo de su imaginación sobre cómo hacer las conexiones, como crear las relaciones, como definir la maternidad, lo que reconfigura la identidad e integra al hijo/a en el seno de las relaciones de parentesco, sin desestabilizarlas (Fitó, 2013).

En relación con la crianza y al cuidado de los hijos/as, las entrevistadas destacan como positivo el hecho de no compartir las decisiones sobre la crianza con una pareja. Algunos ejemplos de los testimonios son los siguientes:

Vos imagínate que el hecho de tener 44 años, que viví sola en este tiempo prácticamente, mis decisiones son mías, me compre el auto yo, elegí yo el modelo, elegí la concesionaria, elijo el color, quise viajar, viaje donde quise no tuve que compartir no que sí, que vamos, que no vamos, viaje sola un mes a Europa, un mes a Estados Unidos, sola, no no no, solo a veces pensarlo lo que podría ser a nivel pareja pensarlo que este no le gustaría estar acá, es aburrido... por eso te digo, que para mí es un placer, entonces estas decisiones que puedo ir tomando para mí son normales. (Cayetana, profesional, 44 años, hija por adopción.)

Asimismo, en los testimonios se observa una fuerte inclinación por no derivar el cuidado de sus hijos/as con terceros más allá de los horarios de jardín y colegio, y lo fundamentan en que concretar la maternidad les llevó tanto tiempo que no lo dejarían en manos de otra persona. Así lo relata Analía: "Porque para tener un hijo y no ocuparme, viste, yo quería ser mama no tener un hijo" (Analía, técnica, 44 años, hija por fertilización asistida).

\section{A modo de cierre}

El objetivo de este trabajo consistió en realizar un análisis inicial sobre la maternidad en solitario como forma de familia contemporánea, describiendo sus particularidades.

El análisis exploratorio del objeto de estudio de la tesis de doctorado, planteado para este trabajo, ha permitido abordarlo desde varios lugares. En primer lugar, desde el estado 
del arte existente hasta el momento, de modo de poder realizar una descripción de la maternidad en solitario y por elección a partir de varias investigaciones encontradas.

Asimismo, se pudo situar en contexto al objeto de estudio en cuestión a partir de plantear un recorrido por las transformaciones que se fueron sucediendo y que han ido modificando la categoría de maternidad como la forma de llevarla a cabo a lo largo de las últimas décadas.

Luego, y en tercer lugar, se realizó un análisis sobre los testimonios recabados en una primera fase de trabajo de campo. Esto ha permitido poder avanzar en algunas puntas de análisis y la emergencia de nuevas hipótesis que guiarán la próxima fase de trabajo de campo para la tesis.

De esta manera, el cierre de este trabajo plantea nuevos interrogantes e hipótesis para continuar con la investigación.

\section{Referencias bibliográficas}

Álvarez-Díaz, J. (2009). ¿La maternidad de un padre o... la paternidad de una madre? Transexualidad, reproducción asistida y bioética. Gac Méd Méx. 146 (2), 151-157. Recuperado de: http://www.medigraphic.com/pdfs/gaceta/gm-2009/gm092l.pdf

Badinter, E. (1991). ¿Existe el instinto maternal? Historia del amor maternal. Siglos XVII al $X X$. Barcelona: Paidós-Pomaire.

Bestard, J. (2011), «Familia y transformaciones en el parentesco», en F. Chacón y J. Bestard (dirs.), Familias. Historia de la sociedad española (del final de la edad media a nuestros días), (pp. 977-1.000). Madrid: Cátedra.

Cadoret. A. (2009), «Mothers for others: between friendship and the market». En Marre y Briggs (eds.). International Adoption. Global Inequalitites and the Circulation of Children. New York: New York University Press.

Beauvoir, S. de. (2012). El Segundo Sexo. Buenos Aires: Debolsillo.

Donoso, S. (2013). Superando la unicidad de la madre: la maternidad lesboparental. En C. Lopez, D. Marre y J. Bestard. (eds.). Maternidades, procreación y crianza en transformación, (pp. 185-202). Barcelona: Bellaterra.

Dorlin, E. (2009). Sexo, género y sexualidad. Introducción a la teoría feminista. Buenos Aires: Nueva Visión.

De Miguel, A. (2000). Los feminismos. En C. Amorós (Dir.). Diez palabras clave sobre la mujer. Pamplona: Verbo Divino. Recuperado de http://acoca2.blogs.uv.es/files/2013/12/Los-feminismos.pdf 
De La Barreda. (2017). Perspectivas biomédicas de la maternidad subrogada. En Cuadernos de Bioética XXVIII 2017/2a http://aebioetica.org/revistas/2017/28/93/153.pdf

Federici, S. (2013). Revolución en punto cero. Trabajo doméstico, reproducción y luchas feministas. Madrid: Traficantes de sueños.

Fitó, C. (2013). Maternidad y paternidad mediante adopción de gametos. Redefiniciones desde la reproducción asistida. En C. López, D. Marre y J. Bestard (eds.). Maternidades, procreación y crianza en transformación, (pp. 19-38). Barcelona: Bellaterra.

Fonseca, C. (1998). Caminos de la adopción. Buenos Aires: Universidad de Buenos Aires.

Friedan, B. (2003). Mi vida hasta ahora. Madrid: Cátedra.

(2009). La mística de la feminidad. Madrid: Cátedra.

Frasquet, R. (2013). La construcción de la maternidad como un proyecto autónomo: el caso de las madres solas por elección a través de técnicas de reproducción asistida en Barcelona. En C. López, D. Marre y J. Bestard (eds.). Maternidades, procreación y crianza en transformación, (pp. 163-183). Barcelona: Bellaterra.

González Rodríguez, M., Jiménez, I.; Morgado, B. y Diez, M. (2004). Madres solas por elección. Análisis de la monoparentalidad emergente. Sevilla: Instituto de la Mujer. Universidad de Sevilla. Recuperado de: http://www.inmujer.gob.es/publicacioneselectronicas/documentacion/Documento s/DE0049.pdf

(2008). Maternidad a solas por elección: primera aproximación. Anuario de Psicología Universitat de Barcelona, (39) 1, 119-126.

Gross, M. (2009), The desire of parenthood among lesbians and gay men, en Marre y Briggs (eds.), International Adoption. Global Inequalitites and the Circulation of Children. New York: New York University Press.

Jociles, M y Rivas, A. (2009). Entre el empoderamiento y la vulnerabilidad: la monoparentalidad como proyecto familiar de las MSPE por reproducción asistida y adopción internacional. En Revista de antropología social 19, 127-170.

-. (2010). ¿Es la ausencia del padre un problema? La disociación de los roles paternos entre las madres solteras por elección. En Gaceta de Antropología, 26 (4). Recuperado de: http://www.ugr.es/ pwlac/G26_04lsabel_JocilesAnaMaria_Rivas.pdf 
Jordana Pröpper, O. (2011). La maternidad voluntariamente sola en Barcelona: una aproximación antropológica. Tesis doctoral. Barcelona: Departamento de Antropología Social, Universitat de Barcelona. Recuperado de: http://diposit.ub.edu/dspace/bitstream/2445/35199/1/OJP_TESIS.pdf

Juliano, D. (2004). Excluidas y marginales. Madrid: Catedra.

Lamas, M. (2002). Cuerpo: diferencia sexual y género. México: Taurus.

Laudano, C. (2013). Shulamith Firestone: Una propuesta pionera acerca del potencial liberador de la tecnología en la vida de las mujeres. III Jornadas del Centro Interdisciplinario de Investigaciones en Género. La Plata: Facultad de Humanidades y Ciencias de la Educación, Universidad Nacional de La Plata. Recuperado de: http://www.memoria.fahce.unlp.edu.ar/trab_eventos/ev.3435/ev.3435.pdf

Lewin, E. (1995), «On the outside looking in: The politics of lesbian motherhood». En F. D. Ginsburg y R. Rapp (eds.). Conceiving the new world order. The Global Politics of Reproduction. (pp. 103-121). Berkeley: University of California Press.

López Pardina, T. (1999). Simone de Beauvoir (1908-1986). Madrid: Ediciones del Orto.

Moncó, B; Jociles, M; Rivas, A (2011). Madres solteras por elección: representaciones sociales y modelos de legitimación. Nueva antropología. México. (24) 74, 73-92. Recuperado de http://www.scielo.org.mx/scielo.php?pid=S018506362011000100004\&script=sci_arttext

Rich, A. (1986). Nacemos de Mujer: La maternidad como experiencia e institución. Valencia: Cátedra.

Sautu, R. (2005). Todo es teoría: objetivos y métodos de investigación. Buenos Aires: Lumiere.

Schwarz, P. (2016). Maternidades en Verbo. Identidades, cuerpos, estrategias, negociaciones: mujeres heterosexuales y lesbianas frente a los desafíos de maternar. Buenos Aires: Biblos.

Smietana, M. (2013). Las paternidades y maternidades en las familias de padres gays creadas por gestación subrogada. En C. López, D. Marre y J. Bestard (eds.). Maternidades, procreación y crianza en transformación (pp. 203-220). Barcelona: Bellaterra.

Stake, R. (1999). Investigación con estudio de caso. Madrid: Morata.

Suárez Llanos, M. (2002). Teoría feminista, política y derecho. Madrid: Dykinson. 
Valles, M. (1999). Técnicas cualitativas de investigación social. Reflexión metodológica y práctica profesional. Madrid: Síntesis.

Varela, N. (2005). Feminismo para principiantes. Barcelona: Ediciones B.

\section{Documento}

Ley $n^{\circ} \mathbf{2 6 . 8 6 2}$ de Acceso integral a los procedimientos y técnicas médico-asistenciales de reproducción médicamente asistida. Buenos Aires: Boletín Oficial, Presidencia de la Nación. 26 de junio de 2013. 